\title{
Incidence of cleft-related speech problems in children with an isolated cleft lip
}

\author{
B. J. A. Smarius ${ }^{1,2} \cdot$ S. Haverkamp ${ }^{3} \cdot$ H. de Wilde ${ }^{3} \cdot$ A. van Wijck-Warnaar ${ }^{4}$ - A. B. Mink van der Molen ${ }^{1,5}$. \\ C. C. Breugem ${ }^{6,7}$
}

Received: 25 March 2019 / Accepted: 21 May 2020 / Published online: 4 June 2020

(C) The Author(s) 2020

\begin{abstract}
Objectives Clinicians agree that children with isolated cleft lip have fewer cleft-associated problems than children with cleft lip and palate. Unfortunately, for isolated cleft lip children, the risk of cleft-associated problems is unknown and maybe underestimated. Often, these children do not get the required follow-up by a multidisciplinary team and thereby not the known benefits in supporting their development. This study examines the incidence of cleft-related speech problems and ear problems in children with isolated cleft lip.

Materials and methods A prospective study was performed on all children born with an isolated cleft lip and treated at the Wilhelmina Children's Hospital in Utrecht between January 2007 and April 2014. Data were collected for sex, date of birth, genetics, cleft lip type, date of cleft lip repair, type of repair, speech/language problems, and ear problems.

Results This study included 75 patients (59\% male). The mean age of the children at the moment of speech examination was 32.5 months (SD 6.1). Eighteen of the 75 children (24\%) needed speech and language therapy; however, only one child (1.3\%) had a cleft-related speech problem. Sixteen of the 75 patients $(21 \%)$ reported a history of one or more episodes of acute otitis media (AOM)/otitis media with effusion (OME) during the first 6 years.

Conclusion/clinical relevance This is the first prospective study analyzing the incidence of cleft-related speech problems in children with an isolated cleft lip. These children do not have a higher risk of cleft-related speech problems or AOM/OME when compared to the general population. However, children with an isolated cleft do have a higher incidence of speech therapy.
\end{abstract}

Keywords Isolated cleft lip $\cdot$ Cleft lip $\cdot$ Cleft-related speech problem $\cdot$ Speech therapy $\cdot$ AOM/OME

\section{Introduction}

The incidence of cleft lip/palate in the Netherlands ranges from 1.4 to 2.1 per 1000 [1]. The inability to close the nasopharynx in case the palate is affected often results in problems with feeding, hearing, speech, and language development [2-4].

Clinicians involved in the care of children with orofacial clefts agree that children with isolated cleft lip have fewer

Electronic supplementary material The online version of this article (https://doi.org/10.1007/s00784-020-03367-5) contains supplementary material, which is available to authorized users.

B. J. A. Smarius

b.j.a.smarius@umcutrecht.nl

1 Department of Pediatric Plastic Surgery, Wilhelmina Children's Hospital, University Medical Center Utrecht, University of Utrecht, Heidelberglaan 100, P.O. BOX 85500, 3508

GA Utrecht, The Netherlands

2 Department of Surgery, Meander Medical Centre, Amersfoort, The Netherlands

3 Speech and Language Therapy, Wilhelmina Children's Hospital, University Medical Center Utrecht, Utrecht, The Netherlands
4 Department of Otorhinolaryngology-Head and Neck Surgery, University Medical Center Utrecht, Utrecht, The Netherlands

5 Department of Plastic Surgery, St. Antonius Hospital, Nieuwegein, The Netherlands

6 Department of Plastic Surgery, Meander Medical Center, Amersfoort, The Netherlands

7 Department of Plastic Surgery, Emma Children's Hospital, University Medical Center Amsterdam, Amsterdam, The Netherlands 
cleft-associated speech and ear problems than children with cleft lip and palate [5]. In most treatment protocols, children with cleft lip and palate are analyzed in a multidisciplinary team. Unfortunately, for children with an isolated cleft lip, the risk of cleft-associated speech and ear problems is unknown and maybe underestimated. It is possible these children do not get the required follow-up by a multidisciplinary team and thereby not the known benefits in supporting their development.

Cleft-related speech problems should be distinguished from non-cleft-related speech problems. Cleft palate patient may be at risk of developing certain deviant speech characteristics, affecting resonance, articulation, and intelligibility, directly caused by the original anomaly and/ or related to incompetent velopharyngeal function.

Velopharyngeal insufficiency (VPI) plays an important role in cleft-related speech problems. VPI is a term used to describe disorders characterized by the abnormal function of the velopharyngeal valve. When the velopharyngeal valve is not functioning correctly, it can cause complications with speech. This can result in several speech disorders, such as hypernasal speech, inability to generate pressure for speech sounds, or the inability to form speech sounds correctly.

Also, the occurrence of recurrent acute otitis media (AOM) and otitis media with effusion (OME) has been reported to be higher in children with cleft palate [6,7]. The etiologic basis for middle ear pathology and hearing loss in patients with cleft palate is considered to be Eustachian tube dysfunction due to functional obstruction, secondary to failure of the palatal muscles to assist in opening the Eustachian tube [8]. Hearing loss in early childhood associated with otitis media with effusion may result in impaired speech, language, and even cognitive development [9].

Vallino et al. concluded that children with isolated cleft lip who develop cleft-associated problems need to be monitored by the multidisciplinary team until all management needs are reached [5].

A multicenter questionnaire study in our hospital investigated the isolated cleft lip population [10]. This retrospective study concluded that isolated cleft lip patients often receive speech and language therapy and these patients should be investigated by the cleft palate team because of the high risk of cleft palate-related symptoms.

Subsequently, since that study, all patients with an isolated cleft lip were prospectively analyzed at the age of 3 years by the cleft team of the University Medical Centre Utrecht (UMCU), the Netherlands.

This study examines the incidence of cleft-related speech and ear problems in children with isolated cleft lip. The study is done to assess whether follow-up is needed in children with isolated cleft lip and gives recommendations regarding follow-up within a team of this specific subgroup of cleft patients.

\section{Methods}

Permission for this study was obtained from the Medical Ethics Review Committee (METC) Board at the University Medical Center, Utrecht, the Netherlands (reference number $\mathrm{WAG} / \mathrm{mb} / 17 / 032751$ ).

All children born with an isolated cleft lip (with or without involvement of the alveolus) and treated at the Wilhelmina Children's Hospital between January 2007 and April 2014 were eligible for this prospective study. All children with any cleft palate involvement were excluded. Data were collected for sex, date of birth, cleft lip type, possible syndromes, date of cleft lip repair, type of repair, speech and language problems, and ear problems.

\section{Definition of isolated cleft lip}

An isolated cleft lip is a cleft that involves the lip only without involvement of the palate. Isolated cleft lip may exist with or without any (complete or incomplete) alveolar cleft. If these structures are partially involved, it is referred to as an incomplete cleft lip. Cleft lip can occur as a one sided (unilateral) or two sided (bilateral). The definition of a cleft lip in this study is an isolated cleft lip with or without involvement of the alveolus.

\section{Cleft lip/palate team care}

It is the standard care at the Wilhelmina Children's Hospital for a specialized multidisciplinary cleft team to analyze all children with all cleft types. Children undergo assessments with a speech and language pathologist, otolaryngologist, pediatric dentist, and plastic surgeon. At the age of 3 years, a speech assessment is a component of the cleft lip/palate program follow-up for isolated cleft lip patients since 2007.

\section{Oral assessment}

At the age of 3 years, the plastic surgeon excluded a cleft palate by visual inspection and if necessary by palpating a possible submucous cleft.

\section{Speech and language assessment}

The speech therapists who work in our cleft team serially assess the speech of patients with a cleft or velopharyngeal dysfunction with other etiology. In the Netherlands, cleft palate patients speech is assessed according The Dutch Cleft Speech Evaluation Test (DCSET) designed for children with orofacial clefts (Supplemental Table: DCSET) [11]. This test has been implemented nationwide in the Netherlands. Speech characteristics in the DCSET are evaluated by the speech 
therapist of our team. The speech therapists in Utrecht do participate in the national calibration sessions for the DCSET.

In our center, the speech therapists assessed all children with isolated cleft lip at the age of 3 years old using the DCSET. Speech was assessed in the following order: resonance, nasal emissions, oral facial muscle function, intelligibility, articulation, and consonant production.

The resonance was subjectively evaluated while the patient speaks loudly 6 nasal, 5 oronasal, and 6 oral sentences. Resonance was sored for each sentence on a 3-point scale. A score of 1 was given for normal resonance, and a score of 3 for severe hypernasality or hyponasality. Nasometry was not used to analyze the resonance because of the insufficient cooperation with the nasometer in young children ( $<4$ years) [12].

Mirror test were performed to detect nasal emissions.

Orofacial muscle function was observed during the assessment. Attention was paid to the following: open mouth, tongue position, and mouth breathing.

The intelligibility was sored during spontaneous speech. Intelligibility was scored on a 5-point scale. A description of the intelligibility scores used by the parents and speech pathologists is presented in Fig. 1.

Finally, articulation was evaluated. Patients were asked to speak aloud words and sentences in a playful way, depending on the age of the patient. If a misarticulation occurred, the type of error was indicated on the form.

\section{Acute otitis media (AOM) and otitis media with effu- sion (OME)}

AOM is one of the most common infections in (early) childhood. It is defined as the presence of middle-ear effusion in conjunction with rapid onset of one or more signs or symptoms of inflammation of the middle ear such as fever, otalgia, and ear discharge (otorrhoea). Middle-ear effusion without signs of an acute infection indicates otitis media with effusion (OME or "glue ear") [13]. The presence or absence of AOM/ OME was determined and documented by the pediatric otolaryngologist. Otitis media with middle ear effusion with consequent conductive hearing loss was an indication for insertion of ventilation tubes. The number of episodes of AOM/
OME and insertion of ventilation tubes was evaluated. If there was a clinical suspicion on hearing loss, the pediatric audiologist conducted hearing tests using an audiometer.

\section{Operation technique}

In this study, all cleft lip surgeries were performed according to the standard treatment protocol at our department and were performed under general anesthesia by two experienced cleft surgeons. Children underwent cleft lip repairs according to Fisher, Tennison, or Mulliken (in bilateral cleft lip) at the age of 3 to 6 months [14-16]. Using these techniques, the cleft repair is combined with paranasal and perioral muscle reconstruction.

\section{Analysis}

Patient characteristics were summarized by descriptive statistics. The Wilcoxon signed-rank test was used for data analysis of the intelligibility scores. All statistical analyses were performed using IBM Statistical Package for Social Science (SPSS) version 22 (SPSS Inc., Chicago, IL, USA). All calculated $P$ values were considered significant if less than 0.05 .

\section{Results}

\section{Characteristics}

This study included 85 patients who underwent only a cleft lip operation between January 2007 and April 2014. No submucous cleft was identified in this subgroup of patients. Ten patients were lost to follow-up or had incomplete followup information. A total of 75 children had complete analyses and were included for analyses. Fifty-nine percent $(n=44)$ of the children were boys. Mean age at cleft lip surgery was 3.6 months (SD 1.1). Patient characteristics are listed in Table 1.

\section{Intelligibility score: The degree of understandability and acceptability}

1. The speech is understandable and normal.

2. The speech differs from others. This does not lead to comments and the speech is understandable.

3. The speech differs from others. This does lead to comments and the speech is understandable.

4. The speech is understandable with some difficulty.

5. The speech is not understandable.

Fig. 1 Intelligibility score: the degree of understandability and acceptability. 1 . The speech is understandable and normal. 2 . The speech differs from others. This does not lead to comments and the speech is understandable. 3. The speech differs from others. This does lead to comments and the speech is understandable. 4. The speech is understandable with some difficulty. 5 . The speech is not understandable 


\section{Genetics and additional anomalies}

Besides the thorough physical examination done in all children, genetic analysis was performed in $7 \%(n=5)$ of the isolated cleft lip patients. An overview of additional anomalies can be found in Table 2.

\section{Isolated cleft lip type}

Seventy-six percent of the children $(n=57)$ had an incomplete cleft lip. Ninety-eight percent of the children $(n=73)$ had a unilateral cleft. Further information of the group is listed in Table 1.

\section{Technique cleft lip closure}

Eighty-three percent of the children were operated according to Fisher $(n=62), 15 \%$ according to Tennison $(n=11)$, and $3 \%$ according to Mulliken $(n=2)$.

\section{Speech and language assessment}

The mean age of the children at the moment of speech examination was 32.5 months (SD 6.1). Eighteen of 75 children (24\%) noticed speech and language problems during the assessment. All those 18 children received speech and language therapy. Three of those 18 children with speech problems had an additional congenital syndrome that most likely influenced the speech development. Exclusion of those 3 children gives an indication for speech and language therapy in $21 \%$ of the children (15/72). Table 3 shows the frequency of occurrence for the features related to speech, language, and hearing in children who needed speech and language therapy.

Table 1 Patient characteristics

\begin{tabular}{ll}
\hline Characteristics & $\begin{array}{l}\text { Patients } \\
n=75(\%)\end{array}$ \\
\hline Gender & \\
Male & $44(59)$ \\
Female & $31(41)$ \\
Age & \\
Mean age at cleft lip repair & 3.6 months (SD 1.1) \\
Mean age at speech assessment & 32.5 months (SD 6.1) \\
Cleft lip type & \\
Incomplete unilateral & $38(51)$ \\
Incomplete unilateral + alveolus involvement & $19(25)$ \\
Complete unilateral & $1(1)$ \\
Complete unilateral + alveolus involvement & $15(20)$ \\
Complete bilateral & $1(1)$ \\
Complete bilateral + alveolus involvement & $1(1)$ \\
\hline
\end{tabular}

Table 2 Patient characteristics

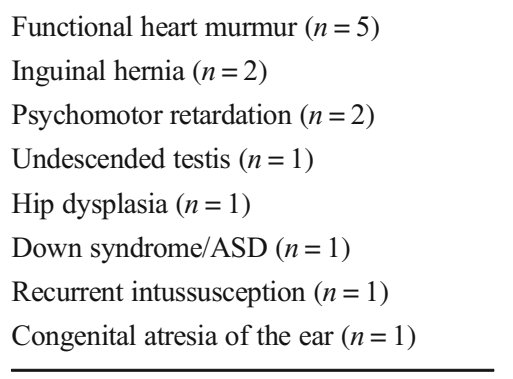

All 18 patients scored a normal resonance during assessment.

There was no nasal emission in one of the 18 patients using the mirror test.

In 10 of the 75 patients (13\%), abnormal orofacial muscle function was observed. In all cases, there was open mouth behavior, which was an indication to start speech and language therapy.

The intelligibility scores evaluated by the speech pathologist and the parents are presented in Table 3. The mean level of intelligibility was 2.2 (range 1-5) and 2.3 (range 1-5) as evaluated by the speech pathologist and parents, respectively. There was no significant difference $(P=0.084)$ in intelligibility score between the speech pathologist and parents, $2.2 \mathrm{vs}$ 2.3 respectively.

The presence of articulation errors was documented in 13 of the 75 patients $(17 \%)$ who needed speech and language therapy. In 1 child $(1 / 75 ; 1.3 \%)$, there was a mild cleftassociated speech problem; this was expressed by interdental speech, palatalization, and assimilation. This patient was treated well with speech and language therapy. A possible submucous cleft was excluded as far as possible by palpating the palate.

\section{Ear problems (AOM/OME)}

Sixteen (21\%) of the 75 patients reported an onset of AOM/ OME. Most children (63\%) reported an onset of AOM/OME between the ages of 0 and 3 years. Sixty-nine percent $(n=11)$ of the children reported a history of 1-3 episodes of AOM/ OME during the first 6 years, while 5 patients had 4 or more episodes (Table 4).

Six of the 16 children (38\%) with a history of AOM/OME received speech and language therapy. Three of those 6 children had 2 or more episodes of AOM/OME.

Eleven children $(69 \%)$ underwent insertion of ventilation tubes because of consequent conductive hearing loss. Three patients underwent two or more ventilation tube insertions (Table 4).

In 1 child, there was a hearing loss as the result of unilateral congenital atresia of the ear; however, there was normal speech development. 


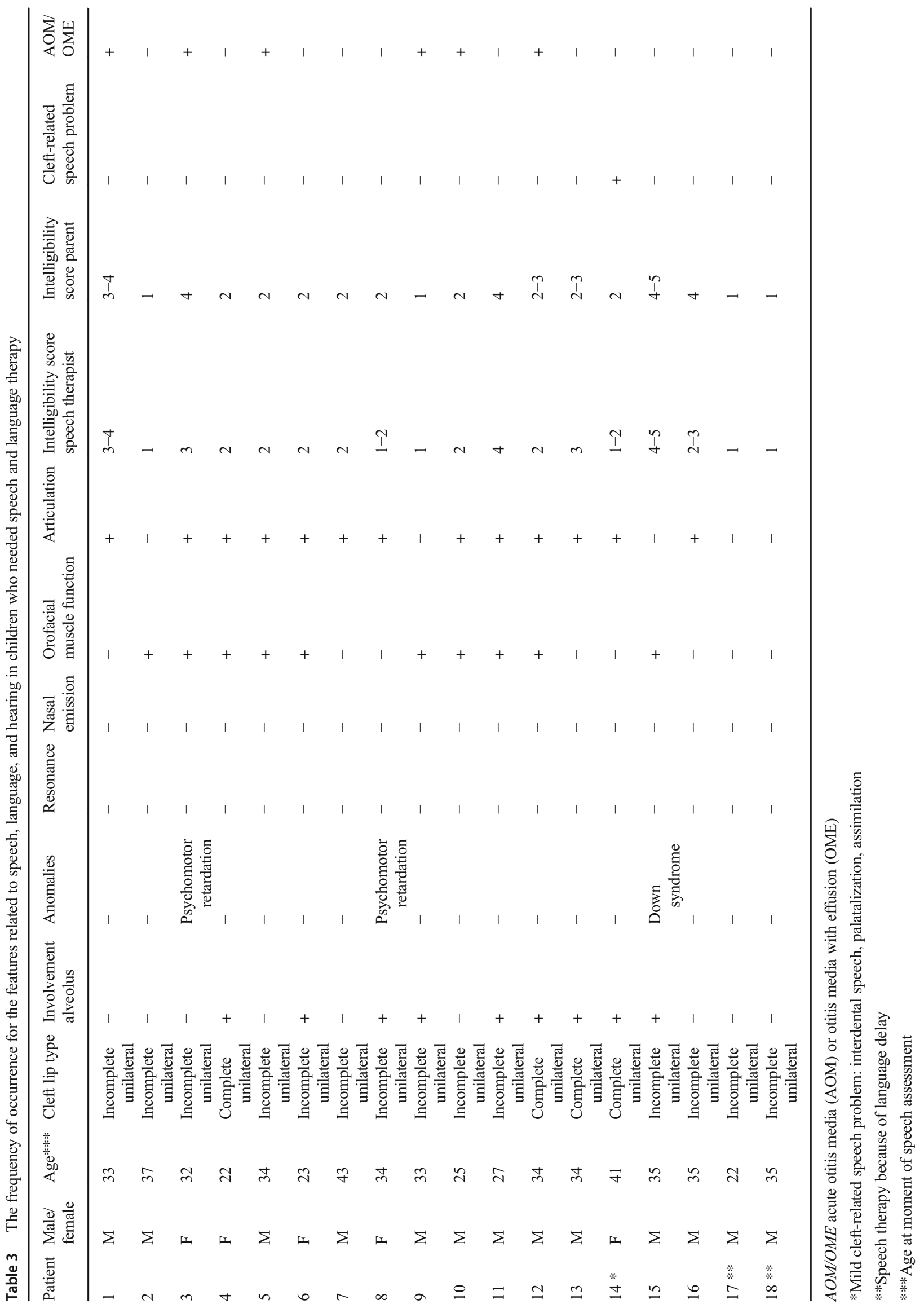




\section{Secondary lip surgery}

In 1 patient (1.3\%), secondary lip surgery was required because of inadequate lip movements. Eleven patients (14.7\%) underwent secondary lip surgery because of cosmetic concerns. All 12 patients who underwent a secondary lip surgery had a unilateral cleft lip. Eleven of the 12 children were treated according to Fisher during primary lip closure. The mean age at secondary cleft lip surgery was 42 months (range 1779 months).

\section{Discussion}

The primary objective in the surgical repair of a cleft lip and/or palate is to achieve an anatomical palatal closure with normal development of speech, hearing, and feeding, while minimizing the possible negative effect of surgery on maxillary outgrowth $[17,18]$. Isolated cleft lip patients have fewer cleftassociated problems than patients with cleft palate involvement [5]. This study provides insights in the development of cleft-associated problems (speech and hearing) in children with isolated cleft lip.

In this study, $24 \%$ of the children needed speech and language therapy. However, $16 \%$ (12/75) of the children were

Table 4 Acute otitis media (AOM)/ Otitis media with effusion (OME) and ventilation tubes

\begin{tabular}{ll}
\hline & Total AOM/OME \\
& $N(\%)$ \\
\hline Number of period AOM/OME & 16 \\
1 time & $7(44)$ \\
$2-3$ times & $4(25)$ \\
$4-5$ times & $2(13)$ \\
$>5$ times & $3(19$ \\
Age of onset AOM/OME & \\
$<1$ year & $2(13)$ \\
$1-3$ years & $8(50)$ \\
$4-6$ years & $5(31)$ \\
$>6$ years & $1(6)$ \\
Number of insertion VT & \\
0 time & $5(31)$ \\
1 time & $8(50)$ \\
$2-3$ times & $3(19)$ \\
Age of onset VT & \\
$<1$ year & \\
$1-3$ years & $0(0)$ \\
$>6$ years & $9(56)$ \\
\hline
\end{tabular}

$V T$ ventilation tubes recorded as having not cleft-associated articulation problems and $0 \%$ had problems with resonance. One child $(1.3 \%)$ was associated with a cleft related articulation problem. Studies of the last 10 years showed comparable outcomes in isolated cleft lip patients (Table 5) [5, 10, 19-27]. However, the reported not cleft-associated speech disorders in this study was higher than in the general population (1-12\%) (Table 5). This can be possible explained by the "extra" follow-up of a multidisciplinary team and the parents' concern in children with an isolated cleft lip. Due to the fact that much attention is paid to the speech development, minor deviations are quickly noticed. Perhaps, those isolated cleft lip children will be referred to a speech and language pathologist more easily/at a lower threshold than children in the general population.

In this study, 1 child (1.3\%) was associated with a cleftrelated speech problem. There are few studies of articulation of children with isolated cleft lip. Riski and DeLong (1984) established that children with isolated cleft lip developed articulation skills that followed a normal developmental schedule [28]. Kono et al. [29] reported that 5\% of the 71 children with cleft lip had velopharyngeal inadequacy and no other visible palatal anomaly [29]. To compare with other clefts, $10-30 \%$ of the primary cleft palate closures still have velopharyngeal insufficiency, and secondary surgery is often imperative [20-22].

Our retrospective study from Deelder et al. concluded that isolated cleft lip patients often receive speech and language therapy compared to the general population [10]. Because of its retrospective nature, we started this prospective study. After this prospective study, we have the same conclusion, but with a $1.3 \%$ cleft-associated speech problem, it seems that isolated cleft lip patients do not have more risk to develop possible velopharyngeal insufficiency than the general population without a cleft lip and/or palate.

Gosain et al. reported "some form of submucous cleft palate" in $36 \%$ of the cleft lip patients. A submucous cleft palate may go unnoticed in isolated cleft lip patients [30]. This could be a reason for those patients to develop cleftassociated problems. All patients in our group had a submucous cleft excluded with physical examination; however, we agree with Gosain et al. to screen all isolated cleft lip patients for a possible submucous cleft palate [30]. Since we had only 1 child with mild VPI that was treated well with speech therapy, we could not demonstrate the suggestion that an associated submucous cleft is common in isolated cleft lip patients.

Recently, Ruegg et al. demonstrated that in non-syndromic CL patients, $31 \%$ had chronic middle ear infections compared to $11 \%$ in the control group [31]. They suggest that an abnormal musculature (missed submucous cleft) could be the cause. In the absence of a cleft palate, the alteration of the shape and size of soft palate, the Eustachian tube itself, the cranial base, or the nasopharyngeal space, could be the cause. However, 
Table 5 Prevalence estimates of articulation and language disorders in preschool children

\begin{tabular}{|c|c|c|c|c|}
\hline Study & Population & Age & $\%$ articulation and language disorders & $\begin{array}{l}\text { Cleft-related speech } \\
\text { problems }\end{array}$ \\
\hline Present study (Smarius et al) & Cleft lip only & 3 years & $\begin{array}{l}\text { Articulation } 17 \% \\
\text { Speech and language therapy } 20 \%\end{array}$ & $1.3 \%$ \\
\hline Vallino et al. [5] & Cleft lip only & 3 years & $\begin{array}{l}\text { Articulation } 13 \% \\
\text { Language } 18 \%\end{array}$ & Not mentioned \\
\hline Deelder et al. (2009) & Cleft lip only & 0.3 to 13.1 years & $\begin{array}{l}\text { Articulation } 15 \% / 22 \% \\
\text { Language } 8 \%\end{array}$ & Not mentioned \\
\hline Tenenbaum [19] & Cleft lip only & 3 to 17 years & Articulation and language $34 \%$ & Not mentioned \\
\hline Witt et al. [20] & All clefts & - & VPI after operation $23 \%$ & $23 \%$ \\
\hline Bicknell et al. [21] & All clefts & - & VPI after operation $25 \%$ & $25 \%$ \\
\hline Mahoney et al. [22] & All clefts & - & VPI after operation $10.3 \%$ & $10.3 \%$ \\
\hline Stevenson and Richman [23] & General population & 3 years & Language $3.1 \%$ & - \\
\hline Silva [24] & General population & 3 years & Language $8.4 \%$ & - \\
\hline $\begin{array}{l}\text { National Institute of Deafness } \\
\text { and other communication } \\
\text { disorders [25] }\end{array}$ & General population & $5-8$ years & Speech sound disorder $8-9 \%$ & - \\
\hline Law et al. $[26]$ & General population & $2-5$ years & Language $5-12 \%$ & - \\
\hline Hannus et al. [27] & General population & $<6$ years & Language 10 per 1.000 & - \\
\hline
\end{tabular}

Ruegg et al. only send questionnaires to parents, while the patients in our group had a physical investigation [31].

Otitis media with effusion (OME) is fluid in the middle ear without signs or symptoms of inflammation. Up to $80 \%$ of children have been affected by OME by the age of 4 years [32]. Acute otitis media (AOM) is characterized by the presence of middle-ear effusion together with an acute onset of signs and symptoms caused by middle-ear inflammation [13]. In high-income countries, the incidence of AOM in children aged 0-5 years ranges from 136 to 273 per 1000 child-years with a peak incidence in the first 2 years of life [33-36]. In the Dutch study from De Hoog et al., 39\% experienced a first AOM before 2 years in the general population [37]. In our study, $21 \%(16 / 75)$ of the isolated cleft lip children in this study reported a history of one or more episodes of AOM/OME during the first 6 years. This is comparable to the $30 \%$ AOM in the study of Deelder et al. [10]. Our study showed a low prevalence of AOM/OME compared to the general population in earlier studies [33-37]. We can conclude that there is no increased risk of AOM in our study population (isolated cleft lip) compared to general population. Thereby, no extra otologic follow-up should be provided to children with isolated cleft lip to prevent hearing loss as a result of recurrent otitis media.

Internationally, the use of different scales to measure speech parameters impedes comparisons of outcomes following treatment [38]. In the Netherlands, the speech of cleft palate patients is assessed according DCSET designed for children with orofacial clefts. For the assessment of the speech in this study, the speech and language pathologist used a subtest of the DCSET because of the young age of the patients (mean age 32.5, SD 6.1). Spruijt et al. measured the inter- and intra-rater reliability of the DCSET providing the possibility of benchmarking the outcome from Dutch cleft lip and palate teams with results from abroad [11]. They concluded that the strength of intra- and inter-rater agreement for most of the parameters was good or very good.

The strength of this study includes the fact that all the patients were operated by two surgeons with a comparable technique and standardized prospective postoperative care and follow-up. All patients with an isolated cleft lip in our hospital are followed up and analyzed at the age of 3 years by the cleft team, and special oral investigations were performed to make sure that no submucous cleft is missed.

In conclusion, this is the first prospective study analyzing the incidence of cleft-related speech problem in children with an isolated cleft lip. These children do not have a higher risk of cleft-related speech problems and AOM/OME than non-cleft children; however, patients with an isolated cleft lip often receive speech and language therapy. We recommend after this study to follow-up isolated cleft lip patients according the local cleft palate protocol but-based on this studythere seems to be no indication for a speech and ear assessment at the age of 3 years old by the speech therapist and otolaryngologist.

\section{Compliance with ethical standards}

Conflict of interest Author BS declares that he has no conflict of interest.

Author SH declares that he has no conflict of interest.

Author HW declares that he has no conflict of interest. 
Author AW declares that he has no conflict of interest.

Author AM declares that he has no conflict of interest.

Author CB declares that he has no conflict of interest.

Ethical approval This article does not contain any studies with human participants or animals performed by any of the authors.

Informed consent For this type of study, formal consent is not required.

Open Access This article is licensed under a Creative Commons Attribution 4.0 International License, which permits use, sharing, adaptation, distribution and reproduction in any medium or format, as long as you give appropriate credit to the original author(s) and the source, provide a link to the Creative Commons licence, and indicate if changes were made. The images or other third party material in this article are included in the article's Creative Commons licence, unless indicated otherwise in a credit line to the material. If material is not included in the article's Creative Commons licence and your intended use is not permitted by statutory regulation or exceeds the permitted use, you will need to obtain permission directly from the copyright holder. To view a copy of this licence, visit http://creativecommons.org/licenses/by/4.0/.

\section{References}

1. Van Den Berg MP, Hage JJ, Kortekaas RTJ, Bloem JJAM (1994) Schisis, een voortdurend probleem voor de patiënt? Ned Tijdschr Geneeskd 138:1058-1061

2. Rohrich RJ, Rowsell AR, Johns DF, Drury MA, Grieg G, Watson DJ, Godfrey AM, Poole MD (1996) Timing of hard palatal closure: a critical long-term analysis. Plast Reconstr Surg 98(2):236-246

3. Sheahan P, Miller I, Sheahan JN, Earley MJ, Blayney AW (2003) Incidence and outcome of middle ear disease in cleft lip and/or cleft palate. Int J Pediatr Otorhinolaryngol 67:785-793

4. Masarei AG, Sell D, Habel A, Mars M, Sommerlad BC, Wade A (2007) The nature of feeding in infants with unrepaired cleft lip and/ or palate compared with healthy noncleft infants. Cleft Palate Craniofac J 44:321-328

5. Vallino LD, Zuker R, Napoli JA (2008) A study of speech, language, hearing, and dentition in children with cleft lip only. Cleft Palate-Craniofacial J 45:485-494

6. Mossey P, Little J (2009) Addressing the challenges of cleft lip and palate research in India. Indian J Plast Surg 42(suppl):9-18

7. IPDTOC Working Group (2011 Jan) Prevalence at birth of cleft lip with or without cleft palate: data from the International Perinatal Database of Typical Oral Clefts (IPDTOC). Cleft Palate Craniofac J 48(1):66-81

8. Bluestone CD, Wittel RA, Paradise JL (1972) Roentgenographic evaluation of Eustachian tube function in infants with cleft and normal palates. Cleft Palate J 9:93-100

9. Teele DW, Klein JO, Chase G, Menyuk P, Rosner BA, Greater Boston Otitis Media Study Group (1990) Otitis media in infancy and intellectual ability, school achievement, speech, and language at age 7 years. J Infect Dis 162:685-694

10. Deelder JD, Breugem CC, de Vries IA, de Bruin M, van der Molen AB M, van der Horst CM (2011 Jun) Is an isolated cleft lip an isolated anomaly? J Plast Reconstr Aesthet Surg 64(6):754-758

11. Spruijt NE, Widdershoven JC, Breugem CC, Speleman L, Homveld IL, Kon M, van der Molen AB M (2012) Velopharyngeal dysfunction and 22q11.2 deletion syndrome: a longitudinal study of functional outcome and preoperative prognostic factors. Cleft Palate Craniofac J 49(4):447-455. https://doi. org/10.1597/10-049
12. van der Heijden P, Hobbel HH, van der Laan BF, Korsten-Meijer AG, Goorhuis-Brouwer SM (2011) Nasometry cooperation in children 4-6 years of age. Int J Pediatr Otorhinolaryngol 75(5):627-630

13. Venekamp RP, Damoiseaux RA, Schilder AG (2014) Acute otitis media in children. BMJ Clin Evid 16:2014

14. Fisher DM (2005) Unilateral cleft lip repair: an anatomical subunit approximation technique. Plast Reconstr Surg 116(1):61-71

15. Tennison CW (1952) The repair of the unilateral cleft lip by the stencil method. Plast Reconstr Surg (1946) 9(2):115-120

16. Kim DC, Mulliken JB (2017) Direct anthropometry of repaired bilateral complete cleft lip: a long-term assessment. Plast Reconstr Surg 140(2):326e-332e

17. Phua YS, de Chalain T (2008) Incidence of oronasal fistulae and velopharyngeal insufficiency after cleft palate repair: an audit of 211 children born between 1990 and 2004. Cleft Palate Craniofac J 45:172-178

18. Losken HW, van Aalst JA, Teotia SS, Dean SB, Hultman S, Uhrich KS (2011) Achieving low cleft palate fistula rates: surgical results and techniques. Cleft Palate Craniofac J 48:312-320

19. Tenenbaum M, Fowler E, Finazzo C, Grames LM, Kane A. Prevalence of speech and language disorders in patients with isolated cleft lip. Presented at the Annual Meeting of the American Cleft Palate-Craniofacial Association; April 2006; Vancouver, British Columbia

20. Witt PD, Wahlen JC, Marsh JL, Grames LM, Pilgram TK (1998) The effect of surgeon experience on velopharyngeal functional outcome following palatoplasty: is there a learning curve? Plast Reconstr Surg 102(5):1375-1384

21. Bicknell S, McFadden LR, Curran JB (2002 Dec) Frequency of pharyngoplasty after primary repair of cleft palate. J Can Dent Assoc 68(11):688-692

22. Mahoney MH, Swan MC, Fisher DM (2013) Prospective analysis of presurgical risk factors for outcomes in primary palatoplasty. Plast Reconstr Surg 132(1):165-171

23. Stevenson J, Richman N (1976 Aug) The prevalence of language delay in a population of three-year-old children and its association with general retardation. Dev Med Child Neurol 18(4):431-441

24. Silva PA (1980) The prevalence, stability and significance of developmental language delay in preschool children. Dev Med Child Neurol 22:768-777

25. National Institute of Deafness and Other Communication Disorders (NIDCD). Statistics on voice, speech, and language. Available at: http://www.nidcd.nih.gov/health/statistics/vs1.asp. Accessed 5 June 2007

26. Law J, Boyle J, Harris F, Harkness A, Nye C (2000) Prevalence and natural history of primary speech and language delay: findings from a systematic review of the literature. Int J Lang Com Dis 35:165188

27. Hannus S, Kauppila T, Launonen K (2009) Increasing prevalence of specific language impairment (SLI) in primary healthcare of a Finnish town, 1989-99. Int J Lang Commun Disord 44(1):79-97

28. Riski JE, DeLong E (1984) Articulation development in children with cleft lip/palate. Cleft Palate J 21:57-64

29. Kono D, Young L, Hotmann B (1981) The association of submucous cleft palate and clefting of the primary palate. Cleft Palate J 18:207-209

30. Gosain AK, Conley SF, Santoro TD, Denny AD (1999) A prospective evaluation of the prevalence of submucous cleft palate in patients with isolated cleftlip versus controls. Plast Reconstr Surg 103(7):1857-1863

31. Ruegg TA, Cooper ME, Leslie EJ, Ford MD, Wehby GL, Deleyiannis FWB, Czeizel AE, Hecht JT, Marazita ML, Weinberg SM (2017 Mar) Ear infection in isolated cleft lip: etiological implications. Cleft Palate Craniofac J 54(2):189-192

32. Williamson I (2011) Otitis media with effusion in children. BMJ Clin Evid 2011:0502 
33. Hak E, Rovers MM, Kuyvenhoven MM, Schellevis FG, Verheij TJ (2006) Incidence of GP-diagnosed respiratory tract infections according to age, gender and high-risk co-morbidity: the Second Dutch National Survey of General Practice. Fam Pract 23:291-294

34. Gribben B, Salkeld LJ, Hoare S, Jones HF (2012) The incidence of acute otitis media in New Zealand children under five years of age in the primary care setting. J Prim Health Care 4:205-212

35. Marchisio P, Cantarutti L, Sturkenboom M, Girotto S, Picelli G, Dona D, Scamarcia A, Villa M, Giaquinto C, Pedianet (2012) Burden of acute otitis media in primary care pediatrics in Italy: a secondary data analysis from the Pedianet database. BMC Pediatr $12: 185$
36. Liese JG, Silfverdal SA, Giaquinto $C$ et al (2014) Incidence and clinical presentation of acute otitis media in children aged $<6$ years in European medical practices. Epidemiol Infect 142:1778-1782

37. de Hoog MLA, Fortanier AC, Smit HA et al (2016) Impact of earlyonset acute otitis media on multiple recurrences and associated health care use. J Pediatr 177:286-291.e1

38. Lohmander A, Olsson M (2004) Methodology for perceptual assessment of speech in patients with cleft palate: a critical review of the literature. Cleft Palate Craniofac J 41:64-70

Publisher's note Springer Nature remains neutral with regard to jurisdictional claims in published maps and institutional affiliations. 